Research Article

Mustafa Özgür Yayli*

\title{
Stability analysis of a rotationally restrained microbar embedded in an elastic matrix using strain gradient elasticity
}

https://doi.org/10.1515/cls-2019-0001

Received Apr 02, 2018; accepted Apr 20, 2018

\begin{abstract}
The buckling of rotationally restrained microbars embedded in an elastic matrix is studied within the framework of strain gradient elasticity theory. The elastic matrix is modeled in this study as Winkler's oneparameter elastic matrix. Fourier sine series with a Fourier coefficient is used for describing the deflection of the microbar. An eigenvalue problem is obtained for buckling modes with the aid of implementing Stokes' transformation to force boundary conditions. This mathematical model bridges the gap between rigid and the restrained boundary conditions. The influences of rotational restraints, small scale parameter and surrounding elastic matrix on the critical buckling load are discussed and compared with those available in the literature. It is concluded from analytical results that the critical buckling load of microbar is dependent upon rotational restraints, surrounding elastic matrix and the material scale parameter. Similarly, the dependencies of the critical buckling load on material scale parameter, surrounding elastic medium and rotational restraints are significant.
\end{abstract}

Keywords: Buckling, microbar, embedded in an elastic matrix, rotational restraints, Fourier sine series

\section{Introduction}

Microbars have been used in NEMS and MEMS (nano, micro electro-mechanical systems). The typical dimensions of microbars are on the order of sub-microns and microns. It is shown in some theoretical and experimental researches that the machines and the structures become

\footnotetext{
^Corresponding Author: Mustafa Özgür Yayli: Uludag University Faculty of Engineering Department of Civil Engineering 16059 Görükle Campüs / BURSA / Turkey;

Email: ozguryayli@uludag.edu.tr; Tel.: +90 22429409 06; Fax: +902242941903
}

ə Open Access. (c) 2019 M. Özgür Yayli, published by De Gruyter. (cc) BY License stiffer in micro sizes [1-3]. It is experimentally shown by Lam et al. [2] in bending test of epoxy microbars that the rigidity of bending stiffness increases about 2.4 times as the vertical thickness of the microbars reduces from 115 to 20 micrometer.

The classical beam theories known as Timoshenko, Euler Bernoulli, Reddy Bickford, which is commonly utilized for studying the buckling, bending, vibration response of beams, does not take account the small size effect. Consequently, these theories leads to less accurate results for smaller values of the material scale parameter to length ratio. The experimental and theoretical results show that classical continuum mechanic theories do not have the ability to predict the small size dependent deformation behavior of micro-sized structures and machines. In order to investigate the micro mechanical response of small sized-structures and machines, several higher order elasticity theories have been proposed such as micropolar theory by Eringen [4], couple stress theory in Ref. [5$7]$, strain gradient theories in $[8,9]$ and nonlocal elasticity theory in $[10,11]$.

According to Eringen's elasticity model, stresses at a point are a function not only of the strains at this point. In the nonlocal elasticity theory proposed by Eringen stresses are also a function of the strains at all points in the domain [11]. This higher order continuum theory is applied for modeling mechanical behaviors of nanobeams, microtubules and carbon nanotubes [12-14]. Reddy and Pang [15] have recently proposed the Euler-Bernoulli and Timoshenko beam theories using the constitutive equations of Eringen [11]. Some of researchers have examined the static bending behaviours of single walled, double and multiwalled carbon nanotubes like [16], [17] and [18]. Pradhan and Murmu [19] have investigated a single nonlocal beam model to determine the dynamic and static characteristics of a nanocantilever beam. Free vibration behavior of carbon nanotubes embedded in an elastic matrix has been explored by some researchers like [20, 21]. Free axial vibration of the nanorods has been investigated by Aydogdu [26]. Dynamic behavior of carbon nanotubes have been 
studied by some researchers like [22-25]. The small size effects on free vibrations of heterojunction single walled carbon nanotubes based on the non-classical and classical beam theories have been studied by Filiz and Aydogdu [27].

Yang et al. [28] have presented a theory known as the modified couple stress theory in which only one length scale parameter is included. In this theory strain energy density includes symmetric rotation gradient tensors besides symmetric strain tensor. Thereafter, these higher order elasticity theories (nonlocal elasticity, strain gradient elasticity and modified couple stress theories) have been widely used to applied the dynamic. static, and buckling analysis of bars and microbars [29-37].

In this study, elastic buckling behavior of size dependent micro-sized beams resting on Winkler type elastic foundation under various types of supporting conditions is explored based on a strain gradient elasticity theory for the first time. It is assumed that the microbeam is simply supported with rotational restraints at the ends. Implementing Stokes' transformation to deformable boundary conditions, a coefficient matrix is obtained and the eigenvalues of this matrix give the buckling loads. Numerical results include comparison with previously published papers are presented for the critical buckling loads which fully demonstrates the accuracy and reliability of the present method. In addition, eigen value results computed analytically are presented to figure out the effects of length scale parameter, rotational restraints and Winkler's parameter on the elastic stability characteristics of the microbar.

\section{Problem definition and modeling}

Consider a simple supported microbar embedded in an elastic matrix with rotational restraints. The microbar is subjected to an in-plane axial load $P$ as shown in Figure 1. The aim of the present study is to investigate small size

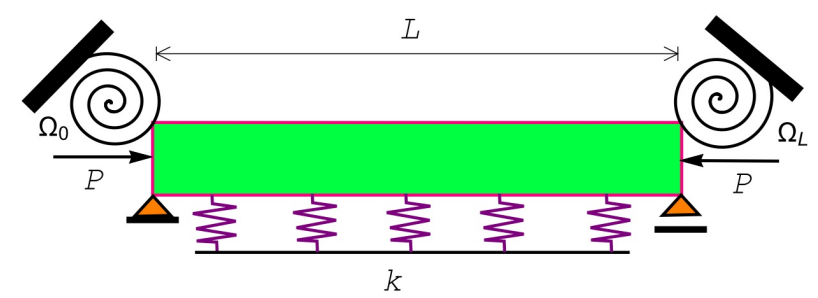

Figure 1: A simple supported microbar embedded in an elastic matrix with rotational restraints. effects on the stability analysis of microbar embedded in an Winkler type elastic matrix. To accomplish this, strain gradient elasticity theory which known as a higher order elasticity theory proposed by Papargyri-Beskou et al. [38] is used in this study. According to gradient elasticity theory, the sixth order governing differential equation of a microbar embedded in an Winkler type elastic matrix is given by [38];

$$
E I\left(\frac{d^{4} w}{\partial z^{4}}-\gamma^{2} \frac{d^{6} w}{\partial z^{6}}\right)+P \frac{d^{2} w}{\partial z^{2}}+k_{w} w=0
$$

where $z$ is an independent variable, $w$ denotes the lateral displacement of the microbeam. $E I$ is the flexural stiffness and $\gamma$ is the material scale parameter. $k_{w}$ represent the constant of the foundation, known as Winkler's constant. In the case of the restrained boundary conditions (deformable boundary conditions), the analytical solution of Eq. (1) is difficult to calculate, so Fourier sine series expansion together with Stokes' transformation will be used for the solution of Eq. (1). The displacement function $w(z)$ is described in three separate regions, two for simply supported ends and the other for the intermediate places between the supporting points:

$$
\begin{gathered}
w(z)=\psi_{0} \quad z=0, \\
w(z)=\psi_{L} \quad z=L, \\
w(z)=\psi(z)=\sum_{j=1}^{\infty} A_{j} \sin \left(\frac{j \pi z}{L}\right) \quad 0<z<L,
\end{gathered}
$$

with

$$
A_{j}=\frac{2}{L} \int_{0}^{L} \psi(z) \sin \left(\frac{j \pi z}{L}\right) d z .
$$

It is not necessary that the Fourier sine series satisfies rotational restraint boundary conditions, as Stokes' Transformation will be used to match the appropriate boundary conditions. It is noted that the two boundary points, $z=0$ and $z=L$, have been excluded in equation (4). Eq. (2) and (3) gives us freedom to choose the displacement function. First derivative of Eq. (4) yields

$$
\psi^{\prime}(z)=\sum_{j=1}^{\infty} \frac{j \pi}{L} A_{j} \cos \left(\frac{j \pi z}{L}\right),
$$

$\psi^{\prime}(z)$ can be written by a Fourier cosine series

$$
\psi^{\prime}(z)=\frac{b_{0}}{L}+\sum_{j=1}^{\infty} b_{j} \cos \left(\frac{j \pi z}{L}\right) .
$$


The coefficients $\left(b_{0}, b_{j}\right)$ in Eq. (7) are given by

$$
\begin{gathered}
b_{0}=\frac{2}{L} \int_{0}^{L} \psi^{\prime}(z) d z=\frac{2}{L}[\psi(L)-\psi(0)], \\
b_{j}=\frac{2}{L} \int_{0}^{L} \psi^{\prime}(z) \cos \left(\frac{j \pi z}{L}\right) d z \quad j=1,2 \ldots,
\end{gathered}
$$

by applying integration by parts

$$
\begin{aligned}
b_{j} & =\frac{2}{L}\left[\psi(z) \cos \left(\frac{j \pi z}{L}\right)\right]_{0}^{L} \\
& +\frac{2}{L}\left[\frac{j \pi}{L} \int_{0}^{L} \psi(z) \sin \left(\frac{j \pi z}{L}\right) d z\right], \\
b_{j} & =\frac{2}{L}\left[(-1)^{j} \psi(L)-\psi(0)\right]+\frac{j \pi}{L} A_{j} .
\end{aligned}
$$

This algorithmic mathematical procedure is known as Stokes' transformation. The sixth derivatives of lateral displacement function $(\psi(z))$ can be obtained as follows:

$$
\begin{aligned}
\frac{d \psi(z)}{d z} & =\frac{\psi_{L}-\psi_{0}}{L} \\
& +\sum_{j=1}^{\infty} \cos \left(\alpha_{j} z\right)\left(\frac{2\left((-1)^{j} \psi_{L}-\psi_{0}\right)}{L}+\alpha_{j} A_{j}\right) \\
\frac{d^{2} \psi(z)}{d z^{2}}= & \\
- & \sum_{j=1}^{\infty} \alpha_{j} \sin \left(\alpha_{j} z\right)\left(\frac{2\left((-1)^{j} \psi_{L}-\psi_{0}\right)}{L}+\alpha_{j} A_{j}\right) \\
\frac{d^{3} \psi(z)}{d z^{3}}= & \frac{\psi_{L}^{\prime \prime}-\psi_{0}^{\prime \prime}}{L} \\
& +\sum_{j=1}^{\infty} \cos \left(\alpha_{j} z\right)\left(\frac{2\left((-1)^{j} \psi_{L}^{\prime \prime}-\psi_{0}^{\prime \prime}\right)}{L}\right. \\
& \left.-\alpha_{j}^{2}\left(\frac{2\left((-1)^{j} \psi_{L}-\psi_{0}\right)}{L}+\alpha_{j} A_{j}\right)\right) \\
\frac{d^{4} \psi(z)}{d z^{4}}= & -\sum_{j=1}^{\infty} \alpha_{j} \sin \left(\alpha_{j} z\right)\left(\frac{2\left((-1)^{j} \psi_{L}^{\prime \prime}-\psi_{0}^{\prime \prime}\right)}{L}\right. \\
& \left.-\alpha_{j}^{2}\left(\frac{2\left((-1)^{j} \psi_{L}-\psi_{0}\right)}{L}+\alpha_{j} A_{j}\right)\right)
\end{aligned}
$$

$$
\frac{d^{5} \psi(z)}{d z^{5}}=\frac{\psi_{L}^{\prime \prime \prime \prime}-\psi_{0}^{\prime \prime \prime \prime}}{L}
$$

$$
\begin{aligned}
+ & \sum_{j=1}^{\infty} \cos \left(z \alpha_{j}\right)\left(\alpha_{j}^{4}\left(\frac{2\left((-1)^{j} \psi_{L}-\psi_{0}\right)}{L}+\alpha_{j} A_{j}\right)\right. \\
& \left.-\frac{2 \alpha_{j}^{2}\left((-1)^{j} \psi_{L}^{\prime \prime}-\psi_{0}^{\prime \prime}\right)}{L}+\frac{2\left((-1)^{j} \psi_{L}^{\prime \prime \prime \prime}-\psi_{0}^{\prime \prime \prime \prime}\right)}{L}\right), \\
\frac{d^{6} \psi(z)}{d z^{6}} & =-\sum_{j=1}^{\infty} \alpha_{j} \sin \left(z \alpha_{j}\right) \\
& \times\left(\alpha_{j}^{4}\left(\frac{2\left((-1)^{j} \psi_{L}-\psi_{0}\right)}{L}+\alpha_{j} A_{j}\right)\right. \\
& \left.-\frac{2 \alpha_{j}^{2}\left((-1)^{j} \psi_{L}^{\prime \prime}-\psi_{0}^{\prime \prime}\right)}{L}+\frac{2\left((-1)^{j} \psi_{L}^{\prime \prime \prime \prime}-\psi_{0}^{\prime \prime \prime \prime}\right)}{L}\right),
\end{aligned}
$$

where

$$
\alpha_{j}=\frac{j \pi}{L} .
$$

By using the above procedure and substituting Eqs. (4), (13), (15) and (17) into Eq. (1), the Fourier coefficients $A_{j}$ will be found as follows:

$$
A_{j}=\frac{2 \alpha_{j}\left(-\tilde{B}_{j}+(-1)^{j}\left(\mathrm{EI} \tilde{\varphi}_{j}+\psi_{L} \tilde{P_{\eta}}\right)+\psi_{0} \tilde{P_{\psi}}\right)}{L \tilde{P}_{\xi}},
$$

where

$$
\begin{gathered}
\tilde{B}_{j}=\gamma^{2} \mathrm{EI} \psi_{0}^{\prime \prime \prime \prime}+\psi_{0}^{\prime \prime}\left(\gamma^{2} \mathrm{EI} \alpha_{j}^{2}+\mathrm{EI}\right), \\
\tilde{\varphi}_{j}=-\gamma^{2} \psi_{L}^{\prime \prime \prime \prime}+\gamma^{2} \psi_{L}^{\prime \prime} \alpha_{j}^{2}+\psi_{L}^{\prime \prime}, \\
\tilde{P}_{\eta}=P-\operatorname{EI} \alpha_{j}^{2}\left(\gamma^{2} \alpha_{j}^{2}+1\right), \\
\tilde{P_{\psi}}=\gamma^{2} \mathrm{EI} \alpha_{j}^{4}+\mathrm{EI} \alpha_{j}^{2}-P, \\
\tilde{P}_{\xi}=\gamma^{2} \mathrm{EI} \alpha_{j}^{6}+\operatorname{EI} \alpha_{j}^{4}+k_{w}-P \alpha_{j}^{2} .
\end{gathered}
$$

In a more general form, the displacement function of a microbeam embedded in an elastic medium having free free ends becomes

$$
\begin{aligned}
w(z) & =\sum_{j=1}^{\infty} \frac{2 \alpha_{j}\left(-\tilde{B}_{j}+(-1)^{j}\left(E I \tilde{\varphi}_{j}+\psi_{L} \tilde{P_{\eta}}\right)+\psi_{0} \tilde{P_{\psi}}\right)}{L \tilde{P}_{\xi}} \\
& \times \sin \left(\alpha_{j} z\right) .
\end{aligned}
$$

It is interesting to note that the equation (25) is degenerated to that of the classical equation wherein the length scale parameter is taken as zero. 


\section{Solution procedure}

A microbar of length $L$ and with rotational restraints at the ends is considered as in Figure 1. It is then seen that the following force boundary conditions can be written by using strain gradient elasticity [38]:

$$
\begin{aligned}
& \Omega_{0} \frac{d \psi}{d z}=E I\left(\psi_{0}^{\prime \prime}-\gamma^{2} \psi_{0}^{\prime \prime \prime \prime}\right), \quad z=0, \\
& \Omega_{L} \frac{d \psi}{d z}=E I\left(\psi_{L}^{\prime \prime}-\gamma^{2} \psi_{L}^{\prime \prime \prime \prime}\right), \quad z=L,
\end{aligned}
$$

where $\Omega_{0}$ and $\Omega_{L}$ express the stiffnesses of the rotational restraints at the ends. Moreover, the higher order boundary conditions are also taken as,

$$
\begin{aligned}
& \psi_{0}=\psi_{0}^{\prime \prime}=0, \quad z=0, \\
& \psi_{L}=\psi_{L}^{\prime \prime}=0, \quad z=L .
\end{aligned}
$$

It is worthy to note that the simply supported boundary conditions are assumed, and therefore, the lateral deflections at the ends $\left(\psi_{0}, \psi_{L}\right)$ are zero, but the higher order moments $\left(E I \psi_{0}^{\prime \prime}-E I \gamma^{2} \psi_{0}^{\prime \prime \prime \prime}, E I \psi_{L}^{\prime \prime}-E I \gamma^{2} \psi_{L}^{\prime \prime \prime \prime}\right)$ are still included in Eqs. (26) and (27). After some mathematical manipulations, the substitution of Eq. (12) and (19) into Eqs. (26), (27) leads to the two homogeneous equations

$$
\begin{aligned}
& \left(-\sum_{j=1}^{\infty} \frac{2 j^{2} \pi^{2} L E I\left(L^{2}+j^{2} \pi^{2} \gamma^{2}\right) \Omega_{0}}{-\pi^{2} j^{2} P L^{4}+k_{w} L^{6}+E I_{[j]}}\right) \psi_{0}^{\prime \prime} \\
& \left(-\sum_{j=1}^{\infty} \frac{2 j^{2}(-1)^{1+j} \pi^{2} L E I\left(L^{2}+j^{2} \pi^{2} \gamma^{2}\right) \Omega_{0}}{-\pi^{2} j^{2} P L^{4}+k_{w} L^{6}+E I_{[j]}}\right) \psi_{L}^{\prime \prime} \\
& +\left(\sum_{j=1}^{\infty} \frac{2 j^{2} \pi^{2} L^{3} E I \Omega_{0}}{-\pi^{2} j^{2} P L^{4}+k_{w} L^{6}+E I_{[j]}}\right) \psi_{0}^{\prime \prime \prime \prime} \\
& +\left(\sum_{j=1}^{\infty} \frac{2(-1)^{1+j} j^{2} \pi^{2} L^{3} E I \Omega_{0}}{-\pi^{2} j^{2} P L^{4}+k_{w} L^{6}+E I_{[j]}}\right) \psi_{L}^{\prime \prime \prime \prime} \\
& =E I\left(\psi_{0}^{\prime \prime}-\gamma^{2} \psi_{0}^{\prime \prime \prime \prime}\right), \\
& \left(-\sum_{j=1}^{\infty} \frac{2 j^{2}(-1)^{1+j} \pi^{2} L E I\left(L^{2}+j^{2} \pi^{2} \gamma^{2}\right) \Omega_{L}}{-\pi^{2} j^{2} P L^{4}+k_{w} L^{6}+E I_{[j]}}\right) \psi_{0}^{\prime \prime}+ \\
& \left(-\sum_{j=1}^{\infty} \frac{2 j^{2} \pi^{2} L E I\left(L^{2}+j^{2} \pi^{2} \gamma^{2}\right) \Omega_{L}}{-\pi^{2} j^{2} P L^{4}+k_{w} L^{6}+E I_{[j]}}\right) \psi_{L}^{\prime \prime} \\
& +\left(\sum_{j=1}^{\infty} \frac{2(-1)^{1+j} j^{2} \pi^{2} L^{3} E I \Omega_{L}}{-\pi^{2} j^{2} P L^{4}+k_{w} L^{6}+E I_{[j]}}\right) \psi_{0}^{\prime \prime \prime \prime}+
\end{aligned}
$$

$$
\begin{aligned}
& \left(\sum_{j=1}^{\infty} \frac{2 j^{2} \pi^{2} L^{3} E I \Omega_{L}}{-\pi^{2} j^{2} P L^{4}+k_{w} L^{6}+E I_{[j]}}\right) \psi_{L}^{\prime \prime \prime \prime} \\
& =E I\left(\psi_{L}^{\prime \prime}-\gamma^{2} \psi_{L}^{\prime \prime \prime \prime}\right),
\end{aligned}
$$

where

$$
E I_{[j]}=\pi^{6} \gamma^{2} j^{6} E I+L^{2} \pi^{4} j^{4} E I,
$$

and the following non-dimensional quantities are defined

$$
\begin{gathered}
\beta=\frac{\gamma}{L}, \\
\tilde{P_{b}}=\frac{P L^{2}}{E I}, \\
K=\frac{k_{w} L^{3}}{E I}, \\
\tilde{\Omega}_{0}=\frac{\Omega_{0} L}{E I}, \\
\tilde{\Omega}_{L}=\frac{\Omega_{L} L}{E I},
\end{gathered}
$$

where $\tilde{\Omega}_{0}$ and $\tilde{\Omega}_{L}$ are the dimensionless stiffnesses of rotational restraints at the ends. $K$ denotes the elastic medium parameter and $\tilde{P_{b}}$ is the buckling parameter. $\beta$ denotes the material scale parameter. Introducing higher order moments at the ends $\mu_{0}^{\prime \prime}, \mu_{L}^{\prime \prime}$ as $\mu_{0}^{\prime \prime}=\psi_{0}^{\prime}-\gamma^{2} \psi_{0}^{\prime \prime \prime \prime}$ and $\mu_{L}^{\prime \prime}=$ $\psi_{L}^{\prime \prime}-\gamma^{2} \psi_{L}^{\prime \prime \prime \prime}$ and eliminating the second order derivatives $\left(\psi_{0}^{\prime \prime}, \psi_{L}^{\prime \prime}\right)$ from Eqs. (30)- (31), the resulting systems of linear equations can be written in non-dimensional form as

$$
\begin{aligned}
& \left(-1-\sum_{j=1}^{\infty} \frac{2 \pi^{2} j^{2} \overline{\Omega_{0}}}{-\pi^{2} j^{2} \tilde{P_{b}}+K+\pi^{6} \beta^{2} j^{6}+\pi^{4} j^{4}}\right) \mu_{0}^{\prime \prime} \\
& +\left(-\sum_{j=1}^{\infty} \frac{2(-1)^{j} \pi^{2} j^{2} \overline{\Omega_{0}}}{-\pi^{2} j^{2} \tilde{P_{b}}+K+\pi^{6} \beta^{2} j^{6}+\pi^{4} j^{4}}\right) \mu_{L}^{\prime \prime}=0 \\
& \left(-\sum_{j=1}^{\infty} \frac{2(-1)^{j} \pi^{2} j^{2} \overline{\Omega_{L}}}{-\pi^{2} j^{2} \tilde{P_{b}}+K+\pi^{6} \beta^{2} j^{6}+\pi^{4} j^{4}}\right) \mu_{0}^{\prime \prime} \\
& +\left(-1-\sum_{j=1}^{\infty} \frac{\left.2 \pi^{2} j^{2} \overline{\Omega_{L}}\right)}{-\pi^{2} j^{2} \tilde{P}_{b}+K+\pi^{6} \beta^{2} j^{6}+\pi^{4} j^{4}}\right) \mu_{L}^{\prime \prime}=0
\end{aligned}
$$

It is worthy to note that if $\bar{\Omega}_{0}$ and $\bar{\Omega}_{L}$ approach zero, these supports degenerate to pinned pinned boundary conditions, while if $\bar{\Omega}_{0}$ and $\bar{\Omega}_{L}$ approach $\infty$ (infinity), these boundary conditions degenerate to clampedclamped ends. Eqs (38), (39) can be shown in a matrix form to be solved for the constants $\left(\mu_{0}^{\prime \prime}, \mu_{L}^{\prime \prime}\right)$ :

$$
\left[\begin{array}{ll}
\Gamma_{11} & \Gamma_{12} \\
\Gamma_{21} & \Gamma_{22}
\end{array}\right]\left[\begin{array}{l}
\mu_{0}^{\prime \prime} \\
\mu_{L}^{\prime \prime}
\end{array}\right]=0,
$$


where

$$
\begin{gathered}
\Gamma_{11}=-1-\sum_{j=1}^{\infty} \frac{2 \pi^{2} j^{2} \overline{\Omega_{0}}}{-\pi^{2} j^{2} \tilde{P_{b}}+K+\pi^{6} \beta^{2} j^{6}+\pi^{4} j^{4}}, \\
\Gamma_{12}=-\sum_{j=1}^{\infty} \frac{2(-1)^{j} \pi^{2} j^{2} \overline{\Omega_{0}}}{-\pi^{2} j^{2} \tilde{P_{b}}+K+\pi^{6} \beta^{2} j^{6}+\pi^{4} j^{4}}, \\
\Gamma_{21}=-\sum_{j=1}^{\infty} \frac{2(-1)^{j} \pi^{2} j^{2} \overline{\Omega_{L}}}{-\pi^{2} j^{2} \tilde{P_{b}}+K+\pi^{6} \beta^{2} j^{6}+\pi^{4} j^{4}}, \\
\Gamma_{22}=-1-\sum_{j=1}^{\infty} \frac{2 \pi^{2} j^{2} \overline{\Omega_{L}}}{-\pi^{2} j^{2} \tilde{P_{b}}+K+\pi^{6} \beta^{2} j^{6}+\pi^{4} j^{4}} .
\end{gathered}
$$

Eq. (40) defines a standard eigenvalue problem. The buckling loads can be calculated as follows.

$$
\left|\Gamma_{\eta v}\right|=0 \quad(\eta, v=1,2) .
$$

Then, the roots of characteristic equation will gives the buckling loads by assigning the different values of $\bar{\Omega}_{0}$ and $\bar{\Omega}_{L}$. It can be seen that this method gives more flexibility in boundary conditions, the characteristic equation for a microbeam with any kinds of boundary conditions (simply supported, clamped-clamped, clamped-pinned, rotationally restrained ends) will be conveniently obtained from a second $(2 \times 2)$ order determinant. It is not necessary to change lateral displacement function for each change in supporting conditions. The main objective of this paper is to propose a general analytical method for the buckling analysis of microbars with different boundary conditions.

\section{Numerical results and discussion}

\subsection{Convergence for rigid boundary conditions}

In this subsection, for the purpose of validation, the present analytical formulation is used to calculate the buckling loads of a microbar where the Winkler elastic matrix parameter is taken as zero. The small scale parameter is taken as $\beta=0, \frac{1}{20}, \frac{1}{10}$ and compare the results with those available in the literature. The effects of the other parameters used in this study on the critical buckling loads are also determined. A computer code is written in a computer program based on the determinant in Eq. (45). It can be seen that the eigenvalues of coefficient matrix in Eq. (40) are sensitive to truncated terms in the series so that a convergence study should be performed to determine the minimum number of terms.

In following validation studies, the critical buckling parameters are computed by Eq. (45). The first 160 terms of the infinite series are utilized in the calculation. The elastic spring coefficients are taken as $\left(\bar{\Omega}_{0}=\bar{\Omega}_{L}=1 \times 10^{-10}\right)$ for simply supported boundary conditions. It can be observed from the Table 1 that when the values of elastic springs are almost zero the analytical results in this study are exactly match with those reported in [39] (see Table 1). In the second validation study, elastic spring parameters are taken as $\left(\bar{\Omega}_{0}=\bar{\Omega}_{L}=1 \times 10^{+10}\right)$ for a microbar with fixed-fixed ends and results are tabulated in Table 2 . By letting $\left(\bar{\Omega}_{0}=1 \times 10^{-10}\right.$ and $\left.\bar{\Omega}_{L}=1 \times 10^{+10}\right)$, Eq. (45) may be used for the fixed-pinned microbar. According to these Tables 2 and 3, present eigenvalue solution is convergent (see Ref.[39]). From these tables it can be concluded that 160 terms of series are sufficient to compute the accurate results.

Table 1: Validation study for a simple supported microbar using strain gradient elasticity theory.

\begin{tabular}{cccc}
\hline & \multicolumn{2}{c}{ S-S microbeam } & $\bar{\Omega}_{0}=\bar{\Omega}_{L}=0$ \\
$\beta$ & Ref. [39] & $\begin{array}{c}\text { Ref. [40] } \\
\sqrt{\tilde{P}}\end{array}$ & $\begin{array}{c}\text { Present } \\
\sqrt{\tilde{P_{b}}}\end{array}$ \\
\hline 0 & 3.1416 & 3.1416 & 3.1416 \\
$\frac{1}{20}$ & 3.1801 & 3.1801 & 3.1801 \\
$\frac{1}{10}$ & 3.2930 & 3.2930 & 3.2930 \\
\hline
\end{tabular}

Table 2: Validation study for a clamped- pinned microbar using strain gradient elasticity theory.

\begin{tabular}{cccc}
\hline & \multicolumn{2}{c}{ C-P microbeam } & $\bar{\Omega}_{0}=1 \times 10^{-9}, \bar{\Omega}_{L}=1 \times 10^{9}$ \\
$\beta$ & Ref. [39] & Ref. [40] & $\begin{array}{c}\text { Present } \\
\sqrt{\tilde{P_{b}}}\end{array}$ \\
\hline 0 & 4.4934 & 4.4887 & 4.4934 \\
$\frac{1}{20}$ & 4.8485 & 4.8294 & 4.8485 \\
$\frac{1}{10}$ & 5.4660 & 5.4337 & 5.4660 \\
\hline
\end{tabular}

Table 3: Validation study for a clamped-clamped microbar using strain gradient elasticity theory.

C-C microbeam $\quad \bar{\Omega}_{0}=\bar{\Omega}_{L}=1 \times 10^{9}$

\begin{tabular}{cccc}
$\beta$ & Ref. [39] & $\begin{array}{c}\text { Ref. [40] } \\
\sqrt{\tilde{P}}\end{array}$ & $\begin{array}{c}\text { Present } \\
\sqrt{\tilde{P_{b}}}\end{array}$ \\
\hline 0 & 6.2831 & 6.2831 & 6.2831 \\
$\frac{1}{20}$ & 7.3165 & 7.2972 & 7.3165 \\
$\frac{1}{10}$ & 9.1264 & 9.0853 & 9.1264 \\
\hline
\end{tabular}


Table 4: The critical buckling loads of restrained microbar under an elastic matrix

\begin{tabular}{|c|c|c|c|c|c|}
\hline \multirow[t]{2}{*}{ Elastic medium parameter } & \multirow[t]{2}{*}{$\bar{\Omega}_{0}=\bar{\Omega}_{L}$} & \multicolumn{4}{|c|}{$\beta$} \\
\hline & & $\frac{1}{25}$ & $\frac{1}{20}$ & $\frac{1}{15}$ & $\frac{1}{10}$ \\
\hline \multirow{7}{*}{$K=10$} & 1 & 14.783 & 14.897 & 15.126 & 15.727 \\
\hline & 5 & 25.566 & 26.113 & 27.031 & 28.790 \\
\hline & 10 & 33.080 & 34.312 & 36.450 & 40.637 \\
\hline & 15 & 37.319 & 39.089 & 42.279 & 48.928 \\
\hline & 20 & 30.954 & 42.109 & 46.100 & 54.850 \\
\hline & 25 & 41.726 & 44.160 & 48.750 & 59.202 \\
\hline & 30 & 42.990 & 45.632 & 50.676 & 62.494 \\
\hline \multirow{7}{*}{$K=30$} & 1 & 16.809 & 16.921 & 17.150 & 17.752 \\
\hline & 5 & 27.524 & 28.078 & 29.009 & 30.789 \\
\hline & 10 & 34.926 & 36.169 & 38.333 & 40.637 \\
\hline & 15 & 39.072 & 40.852 & 44.070 & 50.790 \\
\hline & 20 & 41.638 & 43.801 & 47.816 & 54.850 \\
\hline & 25 & 43.359 & 45.797 & 50.407 & 60.934 \\
\hline & 30 & 44.585 & 47.227 & 52.288 & 64.175 \\
\hline \multirow{7}{*}{$K=50$} & 1 & 18.218 & 18.944 & 19.174 & 19.777 \\
\hline & 5 & 29.479 & 30.040 & 30.985 & 32.787 \\
\hline & 10 & 36.766 & 38.022 & 40.212 & 44.506 \\
\hline & 15 & 40.819 & 42.609 & 45.857 & 52.649 \\
\hline & 20 & 43.316 & 45.486 & 49.527 & 58.431 \\
\hline & 25 & 44.986 & 47.428 & 52.059 & 62.662 \\
\hline & 30 & 46.174 & 48.817 & 53.894 & 65.852 \\
\hline
\end{tabular}

Table 5: Effect of length scale parameters on the critical buckling loads with different elastic medium parameter

\begin{tabular}{|c|c|c|c|c|c|c|}
\hline & $\beta$ & & & & & \\
\hline$K$ & 0 & $\frac{1}{20}$ & $\frac{1}{15}$ & $\frac{1}{10}$ & $\frac{1}{7}$ & $\frac{1}{5}$ \\
\hline 0 & 28.271 & 33.380 & 35.508 & 39.668 & 44.221 & 49.009 \\
\hline 3 & 28.550 & 33.661 & 35.791 & 39.959 & 44.518 & 49.310 \\
\hline 6 & 28.828 & 33.940 & 36.074 & 40.249 & 44.815 & 49.631 \\
\hline 10 & 29.199 & 34.312 & 36.450 & 40.637 & 45.211 & 50.013 \\
\hline 15 & 29.663 & 34.776 & 36.921 & 41.121 & 45.707 & 50.515 \\
\hline 20 & 30.126 & 35.241 & 37.392 & 41.605 & 46.202 & 51.017 \\
\hline
\end{tabular}

\subsection{Effects of deformable boundary conditions}

Comparison of the buckling loads of restrained microbar is given in Table 4. It can be seen from Table 4 that both elastic stiffness and the critical buckling load increase with the increase of the rotational restraint and elastic medium parameters. In order to more clarify the convergence of the buckling loads $\tilde{P_{b}}$, the numerical results for different small scale parameter and elastic medium parameter are listed in Table 5. The rotational spring parameters $\bar{\Omega}_{0}=\bar{\Omega}_{L}=10$ are taken in the mathematical computation. Table 5 indicates that the elastic matrix parameter $K$ has an important influence on the critical buckling loads. It can be seen that the increasing rates of the length scale parameter become faster by increasing in the critical buckling loads.

To investigate the effects of the rotational spring coefficients on the critical buckling parameters, variation of normalized critical buckling parameters with elastic matrix coefficients $(K)$ values are schematically drawn in Figures 2, 3 and 4 for various values of small scale coefficients. Based on the present results in Figures 2-4, the increasing value of the rotational restraint coefficients leads to an increase in the value of buckling load parameter. As expected, the stiffening effect of rotational restraints is to increase the buckling load parameter. It is also seen that the 


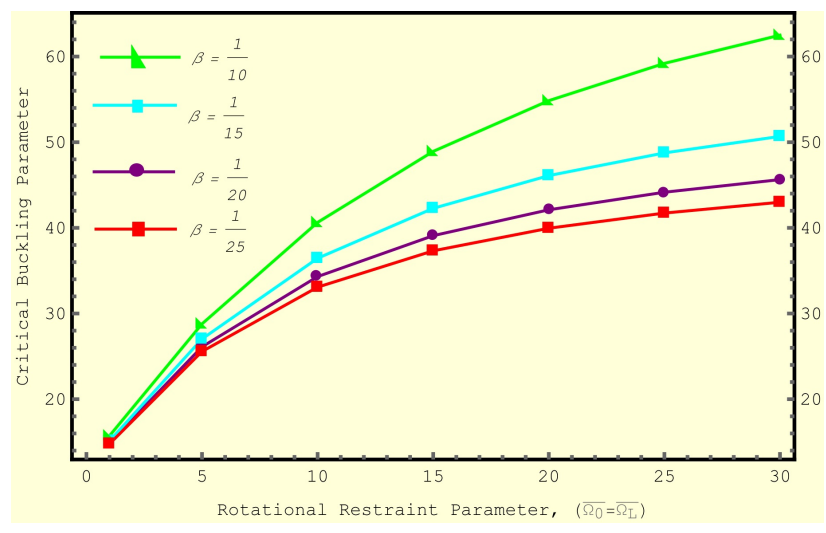

Figure 2: Effects of elastic springs on the critical buckling loads for different small scale parameter with $K=10$

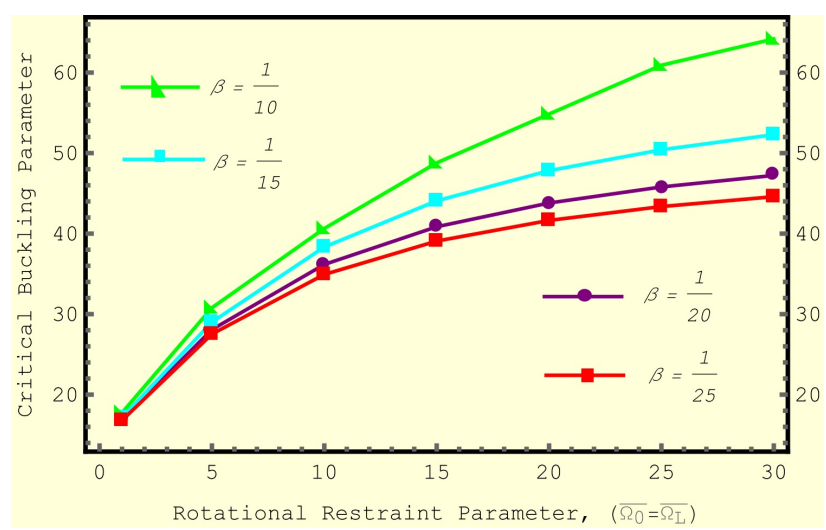

Figure 3: Effects of elastic springs on the critical buckling loads for different small scale parameter with $K=30$

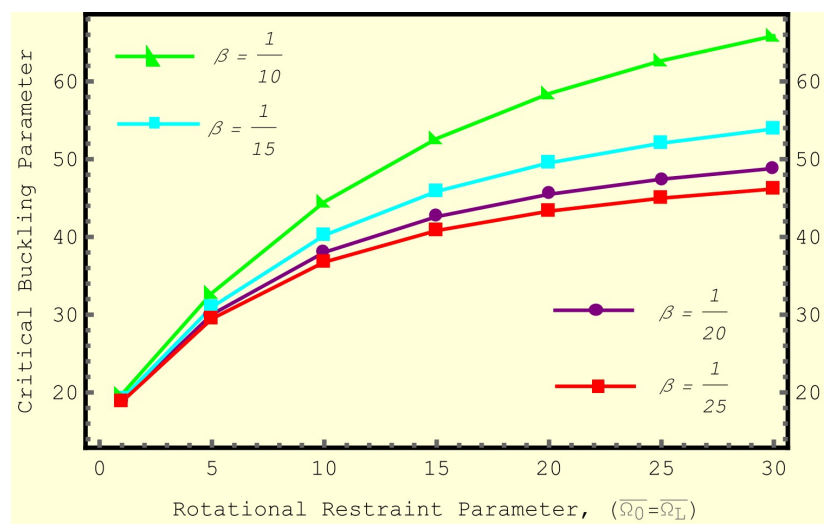

Figure 4: Effects of elastic springs on the critical buckling loads for different small scale parameter with $K=50$

increasing value of length scale parameter $(\beta)$ leads to an increase the critical buckling load.

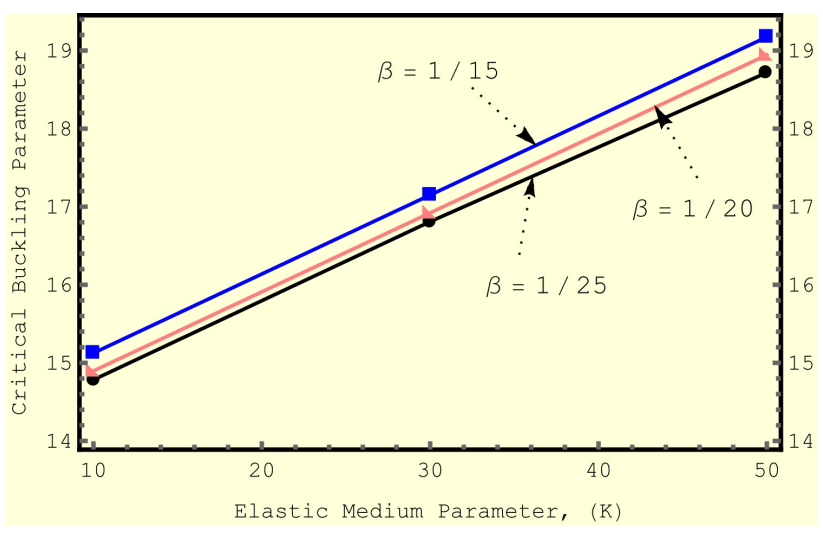

(a) $\bar{\Omega}_{0}=\bar{\Omega}_{L}=1$

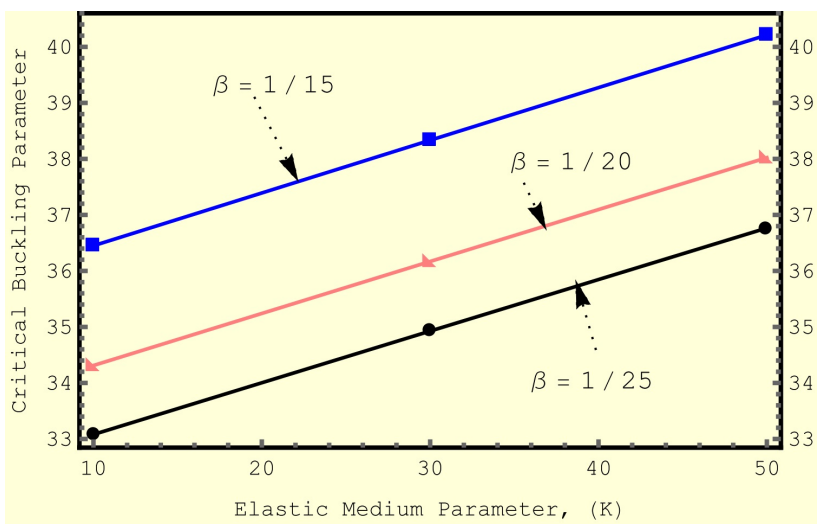

(b) $\bar{\Omega}_{0}=\bar{\Omega}_{L}=10$

Figure 5: Effects of elastic medium parameter on the buckling loads for different small scale parameter

\subsection{Effects of elastic medium parameter}

The effect of the elastic medium parameter on the critical buckling load is demonstrated in Figures 5 This figures display the critical buckling load, as a function of elastic medium parameter for selected rotational spring parameter $\left(\bar{\Omega}_{0}=\bar{\Omega}_{L}=1,10\right)$, and small scale parameter $\beta=1 / 25,1 / 20,1 / 15$ The most important observations are as follows. The differences between the gradient elasticity theory and those of the classical elasticity theory increase, as the small scale parameter increases, especially for the higher rotational spring parameters. As expected, hard rotational springs are more sensitive to small scale effects (see Figures 5).

The effect of the elastic medium parameter on the elastic stability characteristics of the microbeam is also demonstrated in Figures 6. The decreasing value of material scale coefficient leads to an decrease in the magnitude of buckling load. It is also noticed from the Figures 6 . that the decreasing value of the elastic matrix parameter decreases the stiffness of the microbar. 


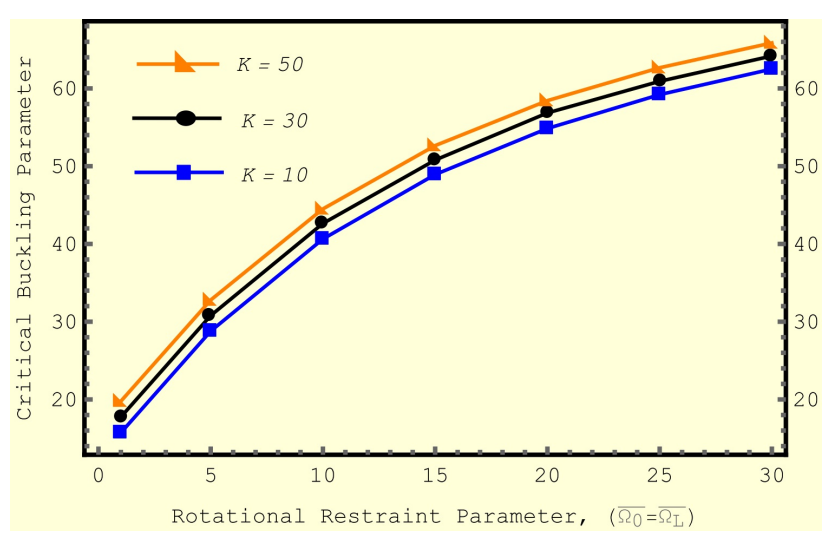

(a) $\beta=1 / 10$

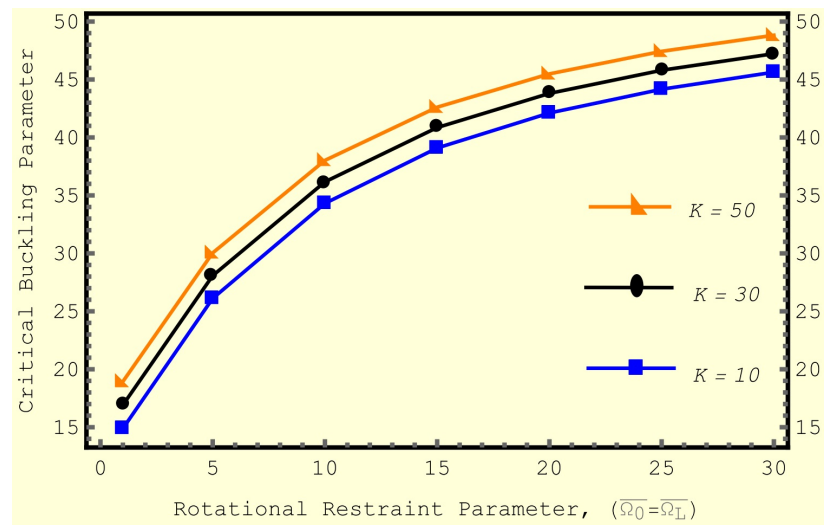

(c) $\beta=1 / 20$

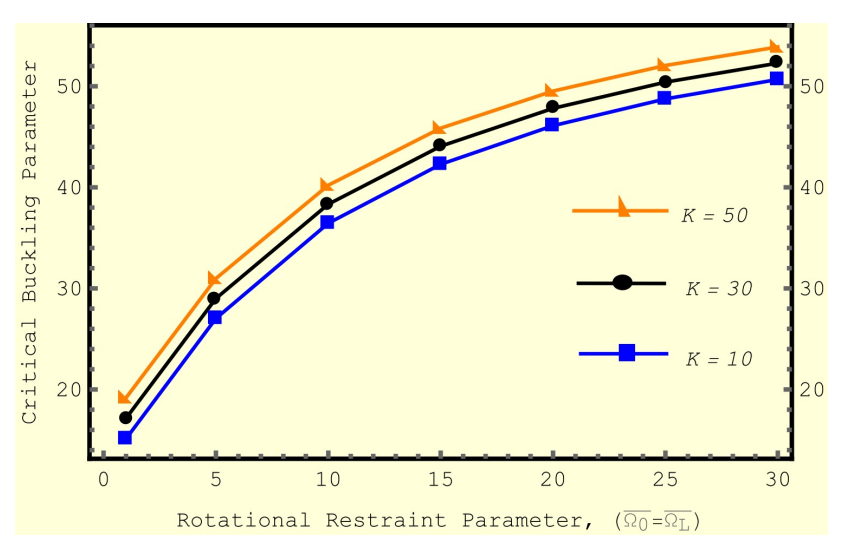

(b) $\beta=1 / 15$

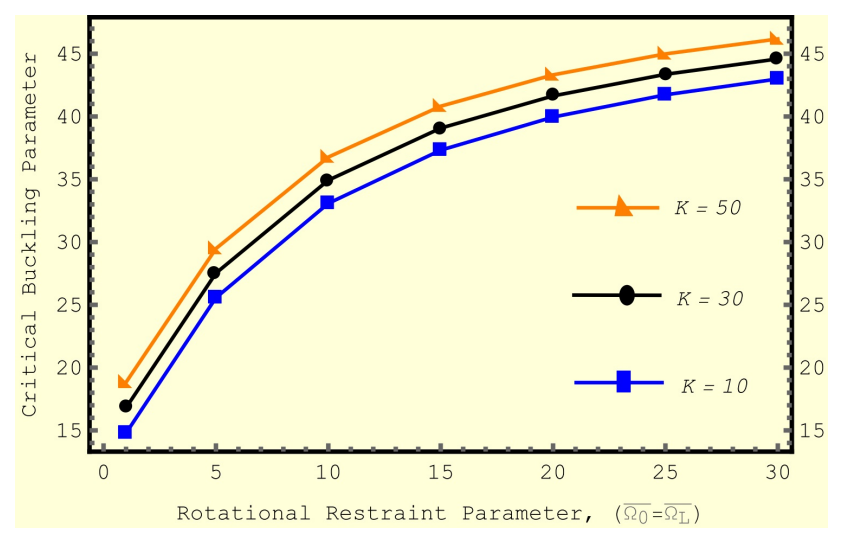

(d) $\beta=1 / 25$

Figure 6: Effects of elastic springs on the critical buckling loads for different elastic medium parameter

\subsection{Effects of small scale parameter}

To illustrate the effect of length scale parameter on buckling responses of microbar, Figure 7 plots schematically the buckling loads with respect to material length scale parameter for a elastically restrained microbeam with equal elastic medium parameter $\bar{\Omega}_{0}=\bar{\Omega}_{L}=10$. The decreasing value of the rotational restraints leads to a decrease in the magnitude of buckling load. It can be also seen that the size effects are more pronounced for larger elastic matrix values $K$ when compared with small ones.

\section{Conclusion}

On the basis of the strain gradient elasticity theory, a useful mathematical approach is proposed for computing the critical buckling loads of restrained microbars embedded in an elastic matrix. The displacement function in the lateral direction is sought as the superposition of Stokes' transformation and a Fourier sine series that is used to

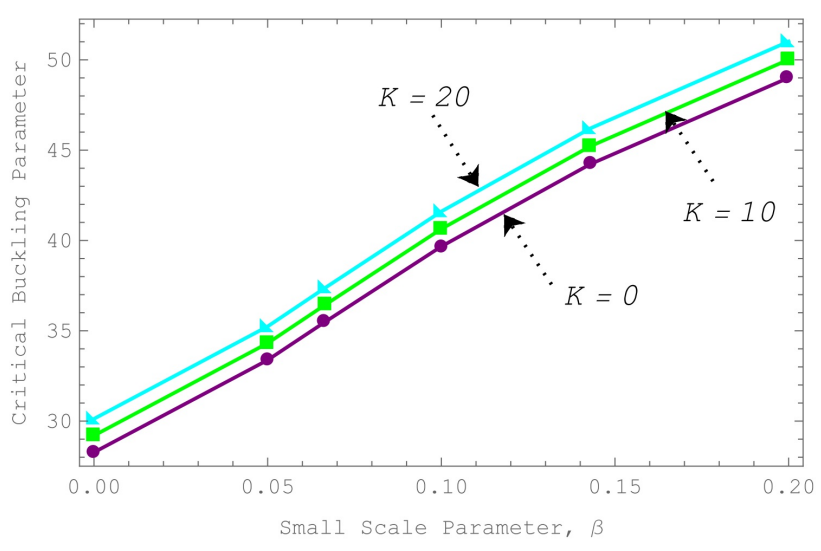

Figure 7: Size effects on the critical buckling loads for different elastic matrix parameter $(K=0,10,20)$

take care of the rotational restraints at the ends. Using the coefficient matrix obtained in present study, the critical buckling parameters of a embedded microbeam with rotationally restrained boundary conditions can be easily computed. The results obtained from published pa- 
pers are used to validate the proposed method which fully demonstrates the reliability and accuracy of the proposed method. The validity of present method is established for simple supported, clamped-clamped and clamped pinned boundary conditions.

Different deformable boundary conditions are considered and solved in same numerical examples. It is indicated that critical buckling loads of microbars are affected by various parameters such as small scale parameter, elastic matrix constant and rotational restraints. It is found that the presence of small size enhances the rigidity of the microbar and increases the critical buckling parameter of microbars. It can be also said that the size effects are more pronounced for higher values of length scale parameter when compared with small ones. With the increasing of boundary constraints, the critical buckling loads of microbars become larger.

\section{References}

[1] Poole, W. J.; Ashby, M. F.; Fleck, N. A. Micro-hardness of annealed and work-hardened copper polycrystals. Scripta Materialia, 1996, 34, 559-564.

[2] Lam, D. C. C.; Yang, F.; Chong, A. C. M.; Wang, J.; Tong. P. Experiments and theory in strain gradient elasticity. J Mech Phys Solids, 2003, 51, 1477-1508.

[3] McFarland, A. W.; Colton, J. S. Role of material microstructure in plate stiffness with relevance to microcantilever sensors., $J$ Micromech Microeng , 2005 15, 1060-1067.

[4] Eringen, A. C. Theory of micropolar plates. Z Angew Math Phys , 1967, 18, 12-30.

[5] Mindlin, R.D.; H. F.Tiersten. Effects of couple-stresses in linear elasticity. ArchRation Mech Anal , 1962, 11, 415-448.

[6] Koiter, W. T. Couple stresses in the theory of elasticity I and II. Proc K Ned Akad Wet (B), 1964, 67, 17-44.

[7] Toupin, R. A. Theory of elasticity with couple stresses.Arch Ration Mech Anal, 1964 17, 85-112.

[8] Fleck, N. A.; Hutchinson, J. W. A phenomenological theory for strain gradient effects in plasticity. J Mech Phys Solids, 1993, 41, 1825-1857.

[9] Fleck, N. A.; Hutchinson, J. W. A reformulation of strain gradient plasticity. J Mech Phys Solids, 2001, 49, 2245-2271.

[10] Eringen, A. C. On differential equations of nonlocal elasticity and solutions of screw dislocation and surface-waves. J. Appl. Phys., 1983, 54, 4703-4710 1983.

[11] Eringen, A. C.; Edelen. D. G. B. On nonlocal elasticity, International Journal of Engineering Science, 1972, 10,233-248.

[12] Sudak, L. J. Column buckling of multiwalled carbon nanotubes using nonlocal continuum mechanics. J. Appl. Phys., 2003, 94, 7281-7287.

[13] Demir, Ç.; Civalek, O. Torsional and longitudinal frequency and wave response of microtubules based on the nonlocal continuum and nonlocal discrete models. Applied Mathematical Modelling, 2013, 37, 9355-9367.
[14] Peddieson, J.; Buchanan, G. R.; McNitt, R. P. Application of nonlocal continuum models to nanotechnology. Int. J. Eng. Sci., 2003, 41, 305-312.

[15] Reddy, J. N.; Pang, S. D. Nonlocal continuum theories of beam for the analysis of carbon nanotubes. Journal of Applied Physics, 2008, 103, 1-16.

[16] Wong, E. W.; Sheehan, P. E.; Lieber, C. M. Nanobeam mechanics: elasticity, strength and toughness of nanorods and nanotubes. Science, 1997, 277, 1971-1975.

[17] Arash, B.; Wang, Q. A review on the application of nonlocal elastic models in modeling of carbon nanotubes and graphenes. Computational Materials Science, 2012, 51, 303-313.

[18] Murmu, T.; Pradhan, S. C. Thermal effects on the stability of embedded carbon nanotubes. Computational Materials Science, 2010, 47, 721-726.

[19] Pradhan, S. C.; Murmu, T. Buckling of single layer graphene sheet based on nonlocal elasticity and higher order shear deformation theory. Physics Letters A, 2010, 373, 4182-4188.

[20] Bachtold, A.; Hadley, P.; Nakanihi, T.; Dekker, C. Logic circuits with carbon nanotube transistors, Science, 2001, 294, 1317-1320.

[21] Kiani, K. A. meshless approach for free transverse vibration of embedded single-walled nanotubes with arbitrary boundary conditions accounting for nonlocal effect.Int J Mech Sci., 2010, 52, 1343-1356.

[22] Ece, M. C.; Aydogdu, M. Nonlocal elasticity effect on vibration of in-plane loaded double-walled carbon nano-tubes. Acta Mech., 2007 190, 185-195.

[23] Simsek, M. Vibration analysis of a single-walled carbon nanotube under action of a moving harmonic load based on nonlocal elasticity theory, Physica-E Low-dimensional Systems and Nanostructures, 2010, 43, 182-191.

[24] Civalek, O.; Demir, C.; Akgöz, B. Free vibration and bending analyses of cantilever microtubules based on nonlocal continuum model, Mathematical and Computational Applications, 2010, 15, 289-298.

[25] Demir, C.; Civalek, O.; Akgöz, B. Free vibration analysis of carbon nanotubes based on shear deformable beam theory by discrete singular convolution Technique, Mathematical and Computational Applications, 2010, 15, 57-65.

[26] Aydogdu, M. Axial vibration of the nanorods with the nonlocal continuum rod model. Physica-E Low-dimensional Systems and Nanostructures, 2009, 41, 861-864.

[27] Filiz, S.; Aydogdu. M. Axial vibration of carbon nanotube heterojunctions using nonlocal elasticity. Computational Materials Science, 2010, 49, 619-627.

[28] Yang, F.; Chong, A. C. M.; Lam, D. C.; Tong, P. Couple stress based strain gradient theory for elasticity. International Journal of Solids and Structures, 2002, 39,2731-2743.

[29] Tornabene, F.: Free vibration analysis of functionally graded conical, cylindrical shell and annular plate structures with a four-parameter power-law distribution. Computer Methods in Applied Mechanics and Engineering, 2009, 198, pp. 2911-2935

[30] Tornabene, F., Viola, E.: Free vibration analysis of functionally graded panels and shells of revolution Meccanica, 2009, 44, pp. 255-281

[31] Tornabene, F., Fantuzzi, N., Bacciocchi, M. Reddy J.N.: An Equivalent Layer-Wise Approach for the Free Vibration Analysis of Thick and Thin Laminated Sandwich Shells Applied Sciences, 2017, 7(17), pp. 1-34 
[32] Tornabene, F., Fantuzzi, N., Bacciocchi, M., Viola, E. and Reddy J.N.: A Numerical Investigation on the Natural Frequencies of FGM Sandwich Shells with Variable Thickness by the Local Generalized Differential Quadrature Method Applied Sciences, 2017, 7(131), pp. 1-39

[33] Park, S. K.; Gao, X. L. Bernoulli-Euler beam model based on a modified couple stress theory. Journal Micromechanics Microengineering, 2006, 16, 2355-2359.

[34] Ma, H. M.; Gao, X. L.; Reddy, J. N. A microstructure dependent Timoshenko beam model based on a modified couple stress theory. Journal of the Mechanics and Physics of Solids, 2008, 56, 3379-3391 2008.

[35] Simsek, M. Dynamic analysis of an embedded microbeam carrying a moving microparticle based on the modified couple stress theory, International Journal of Engineering Science, 2010, 48, 1721-1732.
[36] Yayli, M. Ö. Free vibration behavior of a gradient elastic beam with varying cross section, Shock and Vibration, 2014.

[37] Yayli, M. Ö. A compact analytical method for vibration of microsized beams with different boundary conditions, Mechanics of Advanced Materials and Structures, 2016, (just-accepted), 1-36.

[38] Papargyri-Beskou, S.; Tsepoura, K. G.; Polyzos, D.; Beskos, D. E. Bending and stability analysis of gradient elastic beams, International Journal of Solids and Structures, 2003, 40, 385-400 2003.

[39] Artan, R.; Toksoz, A. Stability analysis of gradient elastic beams by the method of initial value, Arch. Appl. Mech, 2011, 18, 347351.

[40] Yayli, M. O., Stability analysis of gradient elastic microbeams with arbitrary boundary conditions. Journal of Mechanical Science and Technology, 2015, 29, 3373-3380. 